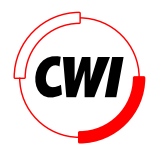

Centrum voor Wiskunde en Informatica REPORT RAPPORT

Parameter estimation in chemical engineering; a case study for resin production

W.J.H. Stortelder

Department of Numerical Mathematics

NM-R9610 July 31, 1996 
Report NM-R9610

ISSN 0169-0388

CWI

P.O. Box 94079

1090 GB Amsterdam

The Netherlands

$\mathrm{CWI}$ is the National Research Institute for Mathematics and Computer Science. CWI is part of the Stichting Mathematisch Centrum (SMC), the Dutch foundation for promotion of mathematics and computer science and their applications.

SMC is sponsored by the Netherlands Organization for Scientific Research (NWO). CWI is a member of ERCIM, the European Research Consortium for Informatics and Mathematics.

Copyright @ Stichting Mathematisch Centrum P.O. Box 94079, 1090 GB Amsterdam (NL) Kruislaan 413, 1098 SJ Amsterdam (NL) Telephone +3120 5929333 Telefax +3120 5924199 


\title{
Parameter Estimation in Chemical Engineering; a case study for resin production
}

\author{
Walter Stortelder \\ CWI \\ P.O. Box 94079, 1090 GB Amsterdam, The Netherlands \\ (e-mail: walterst@cwi.nl)
}

\begin{abstract}
In this report we present a study on parameter estimation in the field of resin production. The mathematical model of the chemical process contains a set of 12 differential algebraic equations (DAEs) and 16 unknown parameters; 8 series of measurements are available, performed under different initial conditions and at different temperatures. To estimate the unknown parameters we solve the system of model equations and tune the model by varying the parameters in order to fit the solution of the DAEs with the measurements.

The differential equations are solved by the BDF method. As a fitness criterion we use the sum of the squared weighted residuals, which is minimised by a Levenberg-Marquardt algorithm.

Not only the optimal parameter values are determined, but also their reliability is investigated in combination with the feasibility of the mathematical model. With the available measured data 12 of the 16 unknown parameters could be estimated within acceptable statistical bounds.
\end{abstract}

AMS Subject Classification (1991): 65C20, 65L08, 93B30.

Keywords and Phrases: Parameter estimation, reaction kinetics, resin production, chemical modelling, model verification, reparameterisation.

Note: This research was supported by the Dutch Technology Foundation (STW) under grant CWI22.2695 


\section{Contents}

1 Introduction 3

2 Reaction Mechanism 3

3 Experiments Performed 3

4 Model Equations 4

5 Treatment of the Melamine Concentrations 6

6 Parameter Estimation 6

$\begin{array}{lll}7 & \text { Numerical Implementation } & 7\end{array}$

8 Statistical Background $\quad 8$

9 Reparameterisation and Results 9

10 Conclusions $\quad 11$

$\begin{array}{ll}\text { References } & 13\end{array}$

Appendix A. The Model File 14 


\section{Introduction}

Parameter estimation can be applied in a wide variety of sciences, such as biology, chemistry, geophysics, electronic engineering, mechanical engineering, etc.. Whenever, besides a model with unknown parameters, a set of corresponding data is given, parameter estimation can be applied. One of the textbooks in this field is [Bar74]. Parameter estimation can be regarded as the inverse of simulation. In simulation the model and the initial conditions are known completely and the future behaviour of the process is investigated by numerical simulation of the model. In case of parameter estimation the governing equations of the model and/or the corresponding initial conditions contain some unknown parameters. On the other hand additional information about the behaviour of the process is given. In the present paper, we consider the situation in which a number of measured data is available.

The model in this paper describes a mechanism of methylolation of melamine by formaldehyde. The methylolation is reversible, nine methylol melamines can be identified. Condensation is not considered. For details on this chemical process we refer to [GHW66]

\section{Reaction Mechanism}

A schematic representation of the chain reactions of interest is given in Figure 1. In this figure we added a label, 'a'-' $k$ ', to every chemical component of interest, formaldehyde is represented by an 'o' and has no label. The explanation of the labels is given in Table 1.

\begin{tabular}{|l|l|l|}
\hline label & abbreviation & full name \\
\hline a & melSol & solid melamine \\
b & melAq & dissolved melamine \\
c & mon & mono-methylol melamine \\
d & di & N,N'-di-methylol melamine \\
e & NN & N,N-di-methylol melamine \\
f & tri & N,N',N'-tri-methylol melamine \\
g & NNN' & N,N,N'-tri-methylol melamine \\
h & tet & N,N,N',N'-tetra-methylol melamine \\
i & NNN'N' & N,N,N',N'-tetra-methylol melamine \\
j & pen & penta-methylol melamine \\
k & hex & hexa-methylol melamine \\
\hline
\end{tabular}

Table 1: Labels, abbreviations and full names of the chemical components.

The reaction mechanism between melamine in solid and dissolved form, labels ' $a$ ' and ' $b$ ' respectively, is unknown. This causes a less straightforward modelling of the process. The adaptations and assumptions we made to overcome this inconvenience will be discussed later.

All other reactions involve the binding and loosening of formaldehyde. The reaction rates which correspond to the binding have a positive subscript. The subscript of a reaction rate is 2 when the binding of formaldehyde is next to another formaldehyde element and 1 otherwise (when the binding is on a free stick of $\lambda$, see Figure 1). Negative subscripts indicate the reverse reaction rates.

For cyclic chemical reaction parts the product of the reaction rates corresponding to the clockwise part should equal the product of the reaction rates anti-clockwise. From the reaction scheme we see that this condition is fulfilled automatically.

\section{Experiments Performed}

As we mentioned before, for parameter estimation we need not only the model with some unknown parameters, but also a set of measured data. Eight series of measurements were performed under 


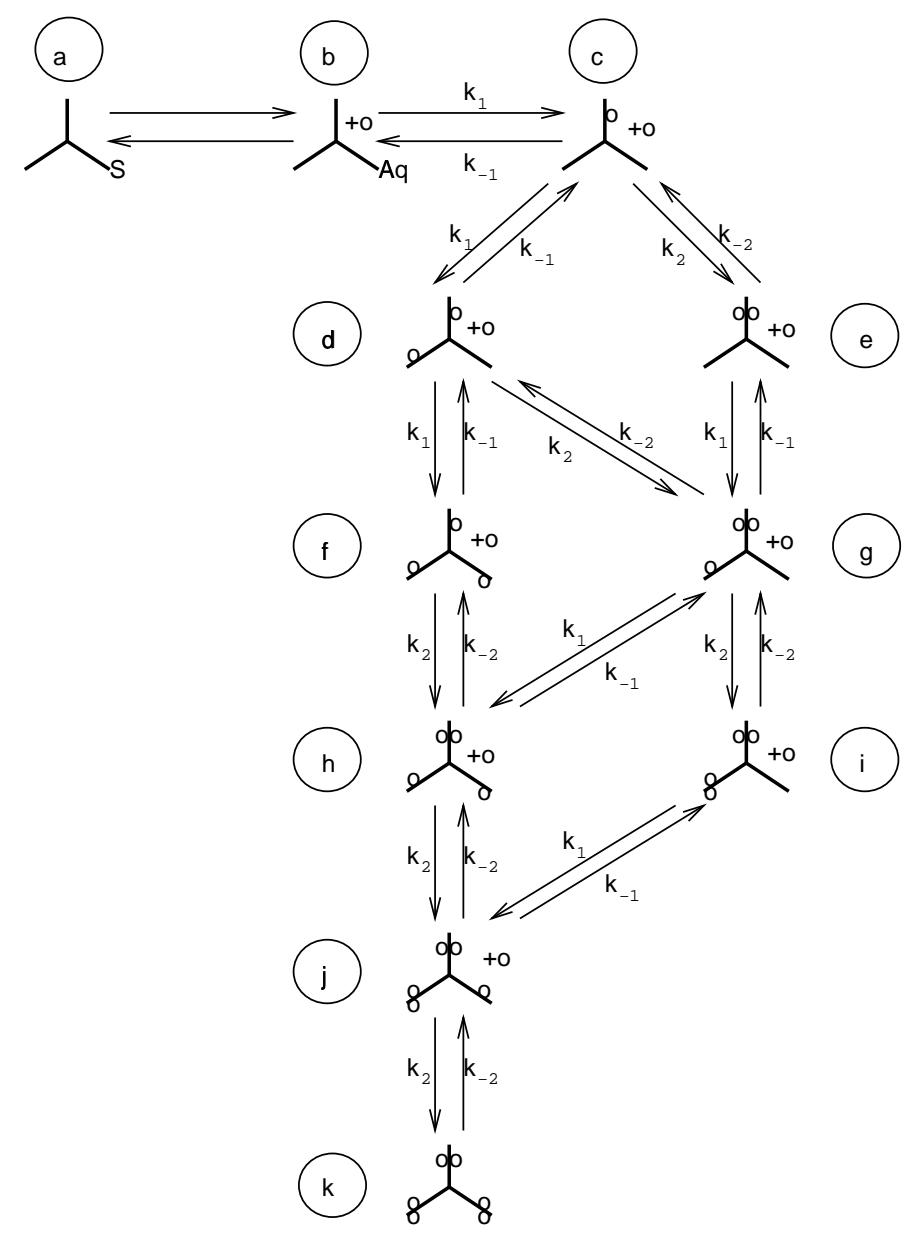

Figure 1: Reaction scheme

different initial conditions and at different temperatures. During each series, at a sequence of times, a sample of the reaction volume was taken, in which the formaldehyde concentration and the concentrations of the components with the labels ' $b$ ' to ' $k$ ' were measured. By measurement we mean the value of the concentration of one chemical component at a specific time, i.e. at each point of time we have 11 measurements. The total number of measurements $(N)$ equals 583 .

\section{Model Equations}

Every differential equation of the mathematical model corresponds to a change in concentration of a chemical species. The derivation of the equations is based on second order reaction kinetics and on conservation of mass.

For illustration we focus on the formation, i.e. the change of concentration per unit of time, of mono-methylol melamine (label ' $c$ ') out of dissolved melamine (label 'b') and formaldehyde. This production depends on $k_{1}$, on the concentrations of formaldehyde and dissolved melamine and on the number of possibilities for the binding of formaldehyde to dissolved melamine. In this case there are six places where the formaldehyde can be bound. The reverse reaction depends on $k_{-1}$, and on the concentration mono-methylol melamine and water. For this case we only have one possibility for the loosening. Following these rules for the reaction kinetics and denoting the formaldehyde concentrations with $[F M]$, the water concentration with $\left[\mathrm{H}_{2} \mathrm{O}\right]$ and the concentration of a methylol melamine by its corresponding abbreviation (see Table 1) inside square brackets, we can derive 
the differential equations for all the species with the labels ' $c$ ' to ' $k$ ', as well as for formaldehyde and water. The resulting differential equations are:

$$
\begin{aligned}
& \frac{\mathrm{d}[F M]}{\mathrm{d} t}=-k_{1}[F M]\left(6[\text { mel } A q]+4[\text { mon }]+2[d i]+4[N N]+2\left[N N N^{\prime}\right]+2\left[N N N^{\prime} N^{\prime}\right]\right)- \\
& k_{2}[F M]\left([m o n]+2[d i]+3[t r i]+\left[N N N^{\prime}\right]+2[\text { tet }]+[\text { pen }]\right)+ \\
& k_{-1}\left[H_{2} \mathrm{O}\right]\left([\text { mon }]+2[d i]+3[\text { tri }]+\left[N N N^{\prime}\right]+2[\text { tet }]+[\text { pen }]\right)+ \\
& k_{-2}\left[H_{2} \mathrm{O}\right]\left(2[N N]+2\left[N N N^{\prime}\right]+2[\text { tet }]+4\left[N N N^{\prime} N^{\prime}\right]+4[\text { pen }]+6[\text { hex }]\right) \text {, } \\
& \frac{\mathrm{d}\left[\mathrm{H}_{2} O\right]}{\mathrm{d} t}=-\frac{\mathrm{d}[F M]}{\mathrm{d} t} \text {, } \\
& \frac{\mathrm{d}[m o n]}{\mathrm{d} t}=6 k_{1}[F M][\text { melAq] }]+2 k_{-1}\left[H_{2} O\right][d i]+2 k_{-2}\left[H_{2} O\right][N N]- \\
& 4 k_{1}[F M][m o n]-k_{2}[F M][m o n]-k_{-1}\left[H_{2} O\right][m o n] \text {, } \\
& \frac{\mathrm{d}[N N]}{\mathrm{d} t}=k_{2}[F M][m o n]+k_{-1}\left[H_{2} O\right]\left[N N N^{\prime}\right]-4 k_{1}[F M][N N]-2 k_{-2}\left[H_{2} O\right][N N] \text {, } \\
& \frac{\mathrm{d}[d i]}{\mathrm{d} t}=4 k_{1}[F M][m o n]+3 k_{-1}\left[H_{2} O\right][t r i]+2 k_{-2}\left[H_{2} O\right]\left[N N N^{\prime}\right]- \\
& 2 k_{1}[F M][d i]-2 k_{2}[F M][d i]-2 k_{-1}\left[H_{2} O\right][d i], \\
& \frac{\mathrm{d}\left[N N N^{\prime}\right]}{\mathrm{d} t}=4 k_{1}[F M][N N]+2 k_{2}[F M][d i]+4 k_{-2}\left[H_{2} O\right]\left[N N N^{\prime} N^{\prime}\right]+ \\
& 2 k_{-1}\left[H_{2} O\right][\text { tet }]-k_{2}[F M]\left[N N N^{\prime}\right]-2 k_{1}[F M]\left[N N N^{\prime}\right]- \\
& 2 k_{-2}\left[\mathrm{H}_{2} \mathrm{O}\right]\left[N N N^{\prime}\right]-k_{-1}\left[\mathrm{H}_{2} \mathrm{O}\right]\left[N N N^{\prime}\right] \text {, } \\
& \frac{\mathrm{d}[t r i]}{\mathrm{d} t}=2 k_{1}[F M][d i]+2 k_{-2}\left[H_{2} O\right][t e t]-k_{2}[F M][t r i]-3 k_{-1}\left[H_{2} O\right][t r i], \\
& \frac{\mathrm{d}\left[N N N^{\prime} N^{\prime}\right]}{\mathrm{d} t}=k_{2}[F M]\left[N N N^{\prime}\right]+k_{-1}\left[H_{2} O\right][\text { pen }]-2 k_{1}[F M]\left[N N N^{\prime} N^{\prime}\right]- \\
& 4 k_{-2}\left[H_{2} O\right]\left[N N N^{\prime} N^{\prime}\right] \text {, } \\
& \frac{\mathrm{d}[\text { tet }]}{\mathrm{d} t}=3 k_{2}[F M][\text { tri }]+2 k_{1}[F M]\left[N N N^{\prime}\right]+4 k_{-2}\left[H_{2} O\right][\text { pen }]- \\
& 2 k_{2}[F M][t e t]-2 k_{-2}\left[H_{2} O\right][t e t]-2 k_{-1}\left[H_{2} O\right][t e t] \text {, } \\
& \frac{\mathrm{d}[\text { pen }]}{\mathrm{d} t}=2 k_{2}[F M][\text { tet }]+2 k_{1}[F M]\left[N N N^{\prime} N^{\prime}\right]-k_{-1}\left[H_{2} O\right][\text { pen }]- \\
& 4 k_{-2}\left[\mathrm{H}_{2} \mathrm{O}\right][\text { pen }]+6 k_{-2}\left[\mathrm{H}_{2} \mathrm{O}\right][h e x]-k_{2}[\text { FM }][\text { pen }] \text {, } \\
& \frac{\mathrm{d}[h e x]}{\mathrm{d} t}=k_{2}[F M][\text { pen }]-6 k_{-2}\left[H_{2} O\right][h e x] \text {. }
\end{aligned}
$$

The concentrations are given in $\mathrm{mol} / \mathrm{kg}$, the time, $t$, in minutes and -hence- all reaction rates, $k_{i}$, in $\mathrm{kg} /(\mathrm{mol} \mathrm{min})$. These reaction rates, which are not known a priori, are the parameters to be estimated. We assume that the change of the reaction volume due to the dissolvation of solid melamine may be neglected.

From the measurements we know that the temperature was not constant over all the experiments. Therefore we account for a temperature dependency in the reaction rates by using Arrhenius' law:

$$
k_{i}(T)=f_{i} e^{\left(\frac{-E_{i}}{R T}\right)}, \quad i \in\{-2,-1,1,2\} \text {. }
$$

Here $f_{i}$ is a pre-exponential factor, $E_{i}$ the activation energy, $R$ the gas constant and $T$ the temperature (in Kelvin). By taking into account this temperature dependency, the number of unknown parameters is doubled.

To solve the set of differential equations, (1)-(11), we need a set of initial conditions. These conditions contain the concentrations of the species of interest at the beginning of an experiment. All the initial concentrations are zero except for water, formaldehyde and dissolved melamine (label 'b'). 


\section{Treatment of the Melamine Concentrations}

We already mentioned that the reaction mechanism between solid and dissolved melamine is unknown. This means that we are not able to derive an equation relating the concentrations of these species. On the other hand the concentration of dissolved melamine appears in the set of differential equations, which means that this concentration is indispensable for solving the differential equations. For each sample taken during the reaction the concentration of dissolved melamine has been determined. To obtain this concentration at the intervening time intervals we used a linear interpolation between the corresponding two subsequent measured concentrations of dissolved melamine.

This leads to a total of 11 differential equations, (1)-(11), and an algebraic equation due to the linear interpolation of the dissolved melamine concentration. The input file for the model equations, as it will be used by the spIds program [EHS95], can be found in Appendix A.

\section{Parameter Estimation}

The resulting system of differential algebraic equations (DAEs) contains, due to the Arrhenius' law, eight unknown parameters $\left(f_{i}\right.$ and $\left.E_{i}\right)$. Besides these unknowns we also do not know the exact initial concentration of formaldehyde for all the series. Because we have eight series of measurements, we get eight extra unknown parameters: $\left[F M_{i}\left(t_{0}\right)\right], i \in\{1, \ldots, 8\}$.

For a convenient shorthand notation we introduce a 16-dimensional parameter vector $\theta$ and a 12-dimensional state vector, $y(t, \theta)$ of varying concentrations, depending on $t$ and $\theta$, as:

$$
\begin{aligned}
& \theta=\left(f_{1}, E_{1}, f_{-1}, E_{-1}, f_{2}, E_{2}, f_{-2}, E_{-2},\left[F M_{1}\left(t_{0}\right)\right],\left[F M_{2}\left(t_{0}\right)\right],\right. \\
& \left.\left[F M_{3}\left(t_{0}\right)\right],\left[F M_{4}\left(t_{0}\right)\right],\left[F M_{5}\left(t_{0}\right)\right],\left[F M_{6}\left(t_{0}\right)\right],\left[F M_{7}\left(t_{0}\right)\right],\left[F M_{8}\left(t_{0}\right)\right]\right)^{T} \text {, } \\
& y=\left([\text { mel } A q],[F M],\left[H_{2} O\right],[m o n],[N N],[d i],\right. \\
& \left.\left[N N N^{\prime}\right],[\text { tri }],\left[N N N^{\prime} N^{\prime}\right],[\text { tet }],[\text { pen }],[\text { hex }]\right)^{T} \text {. }
\end{aligned}
$$

The system of differential algebraic equations and the corresponding initial conditions are now denoted by:

$$
E \frac{d y}{d t}=f(t, y, \theta), \quad y\left(t_{0}, \theta\right)=y_{0}(\theta),
$$

where $E$ is a diagonal, $12 \times 12$ matrix, with $(E)_{11}=0$ and $(E)_{i i}=1$ for $i \in\{2, \ldots, 12\}$. This matrix $E$ accounts for the distinction between differential and algebraic equations. The vector function $f: \mathbb{R} \times \mathbb{R}^{12} \times \mathbb{R}^{16} \rightarrow \mathbb{R}^{12}$ contains the information with respect to the linear interpolation (first component) and the differential equations for $y_{2}, \ldots, y_{12}$ (the righthand sides of (1)-(11)). A measured datum, or shortly: measurement, is one experimentally determined concentration, denoted by the triple:

$$
\left\{\widetilde{y}_{i}, c_{i}, t_{i}\right\}, \quad i \in\{1, \ldots, N\},
$$

with $\widetilde{y}_{i}$ the measured value, $c_{i}$ the measured component, $t_{i}$ the time of the measurement and $N$ the number of measurements.

Finally, we want to estimate the unknown parameter vector, $\theta$, in such a way that the weighted sum of squared discrepancies

$$
S(\theta)=\sum_{i=1}^{N} w_{i}^{2}\left(y_{c_{i}}\left(t_{i}, \theta\right)-\widetilde{y}_{i}\right)^{2}
$$

is minimal. The weights, $w_{i}$, are based on a priori knowledge about the accuracy of the measurements. 


\section{Numerical Implementation}

In order to use a gradient-based minimisation method and to perform some statistical analyses we solve, besides the model equations (13), the sensitivity equations with respect to the unknown parameters. The sensitivity equations, which can be derived analytically from (13) by a computer algebra package, are given by

$$
E \frac{d}{d t} \frac{\partial y}{\partial \theta}=\frac{\partial f}{\partial y} \frac{\partial y}{\partial \theta}+\frac{\partial f}{\partial \theta}, \quad \text { with } \frac{\partial}{\partial \theta} y\left(t_{0}, \theta\right)=\frac{\partial}{\partial \theta} y_{0}(\theta)
$$

Inspection of (16) shows that the Jacobian of the sensitivity equations contains $m$ (the number of unknown parameters) copies of the Jacobian of the original model equations. This means that the sensitivity equations inherit the stiffness character of the original equations. In case of a BDF method (see e.g. [Gea71]), when a certain order and step strategy has been provided for the numerical solution of (13), the same strategy can be used to solve (16) numerically. In this way (16) can be solved very efficiently (see [Hem72]).

To minimise $S(\theta)$ from (15) by Levenberg-Marquardt iterations, we need an initial guess for the unknown parameters, $\theta_{i n i}$. Departing from this initial guess, $\theta=\theta_{i n i}$, we obtain, by numerical integration of (13), the corresponding $N$-dimensional vector of weighted discrepancies

$$
Y(\theta)=w_{i}\left(y_{c_{i}}\left(t_{i}, \theta\right)-\widetilde{y}_{i}\right)_{i=1, \ldots, N} .
$$

Now we can write the sum of squares (15) as

$$
S(\theta)=\|Y(\theta)\|^{2}=Y^{T}(\theta) Y(\theta) .
$$

Simultaneously, the sensitivity equations (16) lead to the calculation of the $N \times m$ matrix

$$
A(\theta)=\frac{\partial Y(\theta)}{\partial \theta}=\left(w_{i} \frac{\partial}{\partial \theta} y_{c_{i}}\left(t_{i}, \theta\right)\right)_{i=1, \ldots, N} .
$$

The minimisation of (18) is done by an iterative procedure. Suppose $\theta$ is a trial vector and its correction is given by $\delta \theta$. The squared sum of the improved parameter vector can be approximated by a quadratic function of $\delta \theta$ as follows

$$
\begin{aligned}
S(\theta+\delta \theta) & =Y^{T}(\theta+\delta \theta) Y(\theta+\delta \theta) \\
& \approx(Y(\theta)+A(\theta) \delta \theta)^{T}(Y(\theta)+A(\theta) \delta \theta) \\
& =Y^{T}(\theta) Y(\theta)+2 \delta \theta^{T} A^{T}(\theta) Y(\theta)+\delta \theta^{T} A^{T}(\theta) A(\theta) \delta \theta .
\end{aligned}
$$

Its minimum is given by the normal equations

$$
A^{T}(\theta) A(\theta) \delta \theta=-A^{T}(\theta) Y(\theta)
$$

where $-A^{T}(\theta) Y(\theta)$ is the descent direction. The last formula is the starting point of a GaussNewton method. It is obvious that the Gauss-Newton procedure becomes unstable if the matrix $A(\theta)$ is (almost) singular. A well known remedy is the use of the Levenberg-Marquardt method, which changes (21) into

$$
\left(A^{T}(\theta) A(\theta)+\lambda I_{m}\right) \delta \theta=-A^{T}(\theta) Y(\theta)
$$

where $\lambda$ is adjusted to the condition of the matrix $A(\theta)$. The Levenberg-Marquardt method can be seen as a combination of Gauss-Newton and steepest descent.

To solve $\delta \theta$ from (22), we use the singular value decomposition (SVD) of the matrix $A(\theta)$,

$$
A(\theta)=U(\theta) \Sigma(\theta) V^{T}(\theta),
$$

where $U(\theta)$ and $V(\theta)$ are $N \times m$ and $m \times m$ matrices, respectively, such that $U^{T}(\theta) U(\theta)=I_{m}$ and $V^{T}(\theta) V(\theta)=V(\theta) V^{T}(\theta)=I_{m}$. The $m \times m$-matrix $\Sigma(\theta)$ is diagonal and contains the singular values in a non-decreasing order. Substitution of (23) into (22) leads to the following expression for the correction of the parameter vector

$$
\delta \theta=-V(\theta)\left(\Sigma^{2}(\theta)+\lambda I_{m}\right)^{-1} \Sigma(\theta) U^{T}(\theta) Y(\theta) .
$$




\section{Statistical Background}

We assume that the errors in the measurements are statistically independent, scaled by the weights, $w_{i}$, in such a way that they have equal variance $\left(\sigma^{2}\right)$ and come from a Gaussian distribution. In case of these reasonable assumptions, the weighted least squares result is identical to the maximum likelihood estimate. The (weighted) experimental errors of the measurements, denoted by $Y(\theta)$ as in (17), have the subsequent covariance matrix:

$$
\mathbf{E}\left(Y(\theta) Y^{T}(\theta)\right)=\sigma^{2} I_{N} .
$$

A combination of this last expression with (21), using the SVD, yields the covariance matrix of $\delta \theta:$

$$
\mathbf{E}\left(\delta \theta \delta \theta^{T}\right)=\sigma^{2}\left(A^{T} A\right)^{-1}=\sigma^{2} V \Sigma^{-2} V^{T} .
$$

Here it is important to note that for the statistical analysis we use (24) with $\lambda=0$. The nonvanishing $\lambda$ is only used in the minimisation process for regularisation. The covariance matrix, evaluated for the final estimate of $\theta$, gives insight into the neighbourhood of the local minimum found.

Upon convergence of the Levenberg-Marquardt algorithm we obtain a final estimate of $\theta$, denoted by $\widehat{\theta}=\theta+\delta \theta$. We can write the corresponding final sum of squares as:

$$
\begin{aligned}
S(\hat{\theta}) & =Y^{T}(\theta+\delta \theta) Y(\theta+\delta \theta) \\
& \approx Y^{T}(\theta) Y(\theta)-\delta \theta^{T}\left(V \Sigma^{2} V^{T}\right) \delta \theta,
\end{aligned}
$$

where the matrices $V$ and $\Sigma$ come from the SVD (see (23)).

Assuming that the linear approximation is valid in the vicinity of $\widehat{\theta}$ and using standard, linear statistics (see [BW88]) we know that $S(\widehat{\theta}) / \sigma^{2}$ and $\delta \theta^{T}\left(V \Sigma^{2} V^{T}\right) \delta \theta / \sigma^{2}$ have a $\chi^{2}$-distribution with $N-m$ and $m$ degrees of freedom respectively. An unbiased estimate of $\sigma^{2}$ is given by

$$
s^{2}=S(\widehat{\theta}) /(N-m) \text {. }
$$

The confidence region at level $\alpha$ is the ellipsoidal region

$$
\delta \theta^{T}\left(V \Sigma^{2} V^{T}\right) \delta \theta \leq \frac{m}{N-m} S(\widehat{\theta}) \mathcal{F}_{\alpha}(m, N-m),
$$

where $\mathcal{F}_{\alpha}(m, N-m)$ is the upper $\alpha$ quantile for Fishers $\mathcal{F}$-distribution with $m$ and $N-m$ degrees of freedom. The independent confidence interval for each estimate is given by:

$$
] \widehat{\theta}_{i}-\delta \theta_{i}^{*}, \widehat{\theta}_{i}+\delta \theta_{i}^{*}[,
$$

with:

$$
\delta \theta_{i}^{*}=\sqrt{\frac{m}{N-m} S(\widehat{\theta}) \mathcal{F}_{\alpha}(m, N-m)\left(V \Sigma^{-2} V^{T}\right)_{i i}} .
$$

Other quantities often used, but only recommended in combination with independent confidence intervals, are the dependent confidence intervals:

$$
] \widehat{\theta}_{i}-\delta \theta_{i}^{\dagger}, \widehat{\theta}_{i}+\delta \theta_{i}^{\dagger}[
$$

with:

$$
\delta \theta_{i}^{\dagger}=\sqrt{\frac{m}{N-m} \frac{S(\widehat{\theta}) \mathcal{F}_{\alpha}(m, N-m)}{\left(V \Sigma^{2} V^{T}\right)_{i i}}} .
$$

The reader is referred to Figure 2 for a graphical interpretation. The principal axes of the ellipsoidal confidence region coincide with the columns of the matrix $V$. The distance from the origin to the ellipse along the $i$-th column of $V$ is proportional to the reciprocal of the $i$-th singular value. This means that small singular values give rise to an elongated confidence region in the corresponding direction. The independent confidence interval of the $i$-th parameter (30) coincides with the projection of the ellipsoidal region on the $i$-th parameter axis, its intersection yields the dependent confidence interval (31). 


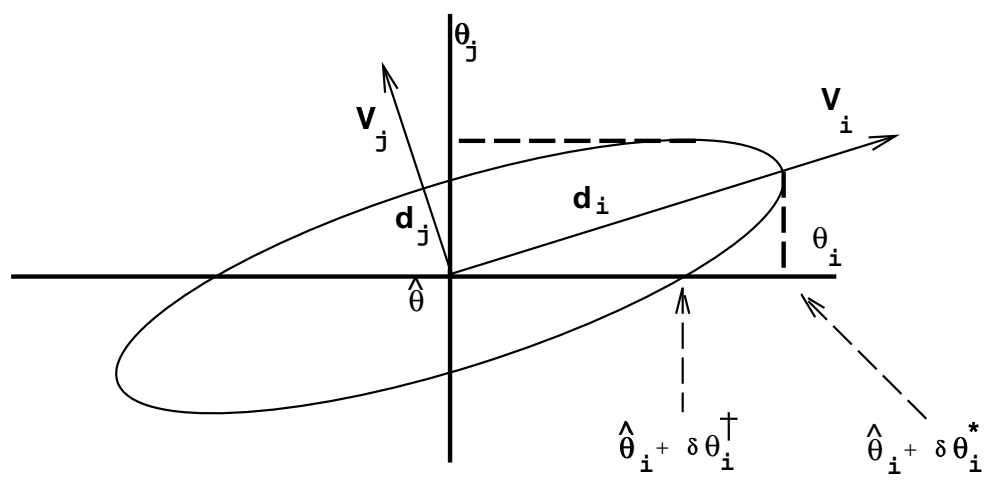

Figure 2: Graph of a 2-dimensional intersection of the ellipsoidal region from (29), centred at $\widehat{\theta}$.

\section{Reparameterisation and Results}

The initial estimates for the pre-exponential factors and the activation energies (based on literature [GHW66]) and the initial formaldehyde concentrations (given by the experimentalists) are listed in Table 2. For numerical reasons it is better to have the parameters of the same order of magnitude.

\begin{tabular}{|l|l|l|l|}
\hline parameter & \multicolumn{1}{|c|}{ value } & parameter & value \\
\hline$f_{1}$ & $1.35 \times 10^{14}$ & $F M_{1}\left(t_{0}\right)$ & 8.41 \\
$E_{1}$ & $9.8 \times 10^{4}$ & $F M_{2}\left(t_{0}\right)$ & 7.61 \\
$f_{-1}$ & $3.98 \times 10^{8}$ & $F M_{3}\left(t_{0}\right)$ & 5.60 \\
$E_{-1}$ & $6.8 \times 10^{4}$ & $F M_{4}\left(t_{0}\right)$ & 5.58 \\
$f_{2}$ & $1.66 \times 10^{15}$ & $F M_{5}\left(t_{0}\right)$ & 4.80 \\
$E_{2}$ & $1.2 \times 10^{5}$ & $F M_{6}\left(t_{0}\right)$ & 4.81 \\
$f_{-2}$ & $8.91 \times 10^{9}$ & $F M_{7}\left(t_{0}\right)$ & 4.80 \\
$E_{-2}$ & $9.0 \times 10^{4}$ & $F M_{8}\left(t_{0}\right)$ & 5.58 \\
\hline
\end{tabular}

Table 2: Initial estimates of the unknown parameters.

To obtain this we take the natural logarithm of the pre-exponential factors, $f_{i}$, and the activation energies are scaled by a factor $1 / 1000, E_{i}^{*}=E_{i} / 1000$. The scaled initial parameter estimates are listed in the second column of Table 3.

After the above mentioned scaling, the first numerical tests were performed. The results are reported in Table 3 . The corresponding graphs of the calculated concentrations and the measured values of $N, N$ ', $N$ "-tri-methylol melamine (label ' $f$ ' in Figure 1) during the second experiment and penta-methylol melamine (label ' $\mathrm{j}$ ') during the eighth experiment for the initial and final parameter values are shown in Figure 3.

The results from Table 3, with respect to the sum of squares and the corresponding graphs are satisfactory; the numerical solution fits the measurements within reasonable bounds. However, the confidence regions for the pre-exponential factors and the activation energies are unsatisfactory. Inspection of the singular values shows that four of them are extremely small. The corresponding eigenvectors, the last four columns of $V$, can be identified with pairs $\left\{\ln \left(f_{i}\right), E_{i}^{*}\right\}$, for $i \in\{-2,-1,1,2\}$. The same holds for the four largest singular values. This means that an intersection of the ellipsoidal region with the $\left\{\ln \left(f_{i}\right), E_{i}^{*}\right\}$-plane gives an elongated ellipse, of which the principal axes are rotated with respect to the coordinate axes. The presence of elongated ellipsoidal regions can also be seen from the quotients of the independent and dependent confidence regions. This indicates that for each pair $\left\{\ln \left(f_{i}\right), E_{i}^{*}\right\}$, only one parameter can be estimated accurately after an appropriate reparameterisation of either $\ln \left(f_{i}\right)$ or $E_{i}^{*}$.

A well known reparameterisation for the pre-exponential factor (see [BDB86, Wat94]) is given 


\begin{tabular}{|l|c|c|c|c|}
\hline & $\begin{array}{l}\text { initial } \\
\text { est. } \\
\left(\theta_{\text {ini }}\right)\end{array}$ & $\begin{array}{l}\text { final } \\
\text { est. } \\
(\widehat{\theta})\end{array}$ & $\begin{array}{l}\text { independent } \\
\text { confidence } \\
\text { regions }\left(\delta \theta^{*}\right)\end{array}$ & $\begin{array}{l}\text { dependent } \\
\text { confidence } \\
\text { regions }\left(\delta \theta^{\dagger}\right)\end{array}$ \\
\hline $\ln \left(f_{1}\right)$ & 32.54 & 20.17 & 5.12 & 0.0728 \\
$E_{1}^{*}$ & 98.00 & 65.38 & 14.0 & 0.198 \\
$\ln \left(f_{-1}\right)$ & 19.80 & 24.81 & 20.5 & 0.469 \\
$E_{-1}^{*}$ & 68.00 & 91.27 & 57.7 & 1.32 \\
$\ln \left(f_{2}\right)$ & 35.05 & 14.17 & 21.8 & 0.261 \\
$E_{2}^{*}$ & 120.00 & 51.03 & 59.7 & 0.717 \\
$\ln \left(f_{-2}\right)$ & 22.91 & 9.126 & 32.2 & 0.407 \\
$E_{-2}^{*}$ & 90.00 & 47.61 & 88.4 & 1.13 \\
$F M_{1}$ & 8.41 & 8.745 & 0.622 & 0.582 \\
$F M_{2}$ & 7.61 & 8.536 & 0.609 & 0.578 \\
$F M_{3}$ & 5.6 & 5.097 & 0.607 & 0.604 \\
$F M_{4}$ & 5.58 & 6.098 & 0.712 & 0.701 \\
$F M_{5}$ & 4.8 & 4.671 & 0.766 & 0.760 \\
$F M_{6}$ & 4.81 & 4.724 & 0.768 & 0.752 \\
$F M_{7}$ & 4.8 & 5.383 & 0.694 & 0.686 \\
$F M_{8}$ & 5.58 & 6.065 & 0.702 & 0.683 \\
\hline$S(\theta)$ & 336.6 & 14.76 & & \\
\hline \multicolumn{5}{|l}{}
\end{tabular}

Table 3: Initial estimates and final estimates of $\theta$ plus statistics.
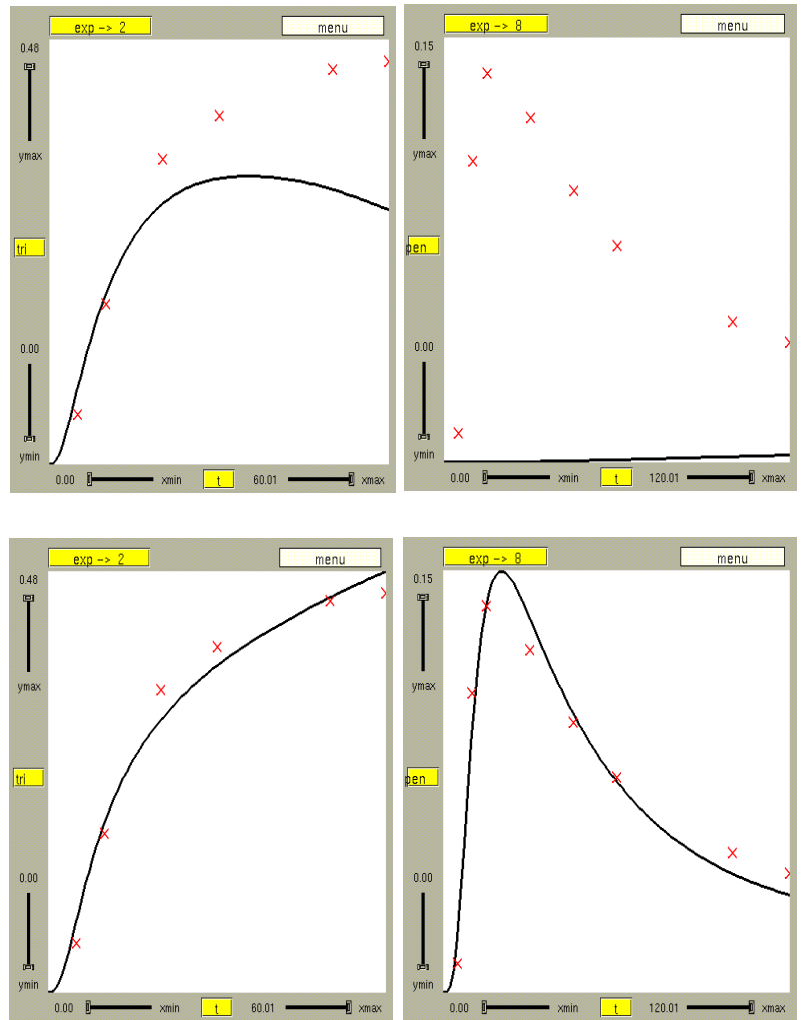

Figure 3: Measured (' $\mathrm{x}$ ') and calculated (solid line) concentrations of $N, N$ ',N"'tri-methylol melamine (label ' $f$ ') during the second experiment (left half) and the penta-methylol melamine (label ' $j$ ') during the eighth experiment (right half), for the initial (upper half) and final (lower half) parameter values from Table 3. 
by the expression:

$$
\begin{aligned}
k_{i}(T) & =f_{i} \exp \left(\frac{-E_{i}}{R T}\right) \\
& =f_{i}^{*} \exp \left(\frac{-E_{i}}{R}\left(\frac{1}{T}-\frac{1}{T_{0}}\right)\right), \quad i \in\{-2,-1,1,2\},
\end{aligned}
$$

with:

$$
f_{i}^{*}=f_{i} \exp \left(\frac{-E_{i}}{R T_{0}}\right) .
$$

The temperature $T_{0}$ is the reference temperature and should be close to the temperatures during the experiments. An appropriate choice for $T_{0}$ is the averaged temperature over all the performed experiments. Note that the reparameterised pre-exponential factors, $f_{i}^{*}$, represent the reaction rates, $k_{i}$, at $T=T_{0}$. The results after this reparameterisation are given in Table 4 for $T_{0}=333 \mathrm{~K}$.

\begin{tabular}{|l|c|l|c|c|}
\hline & $\begin{array}{l}\text { initial } \\
\text { estimates } \\
\left(\theta_{\text {ini }}\right)\end{array}$ & $\begin{array}{l}\text { final } \\
\text { estimates } \\
(\widehat{\theta})\end{array}$ & $\begin{array}{l}\text { independent } \\
\text { confidence } \\
\text { regions }\left(\delta \theta^{*}\right)\end{array}$ & $\begin{array}{l}\text { dependent } \\
\text { confidence } \\
\text { regions }\left(\delta \theta^{\dagger}\right)\end{array}$ \\
\hline $\ln \left(f_{1}^{*}\right)$ & -2.74 & -3.376 & 0.134 & 0.073 \\
$E_{1}^{*}$ & 98.00 & 65.33 & 14.0 & 7.38 \\
$\ln \left(f_{-1}^{*}\right)$ & -4.68 & -8.047 & 0.65 & 0.467 \\
$E_{-1}^{*}$ & 68.00 & 91.91 & 57.2 & 38.3 \\
$\ln \left(f_{2}^{*}\right)$ & -8.15 & -4.181 & 0.621 & 0.261 \\
$E_{2}^{*}$ & 120.00 & 54.23 & 61.4 & 25.1 \\
$\ln \left(f_{-2}^{*}\right)$ & -9.49 & -7.986 & 0.893 & 0.405 \\
$E_{-2}^{*}$ & 90.00 & 53.03 & 88.9 & 38.2 \\
$F M_{1}$ & 8.41 & 8.743 & 0.621 & 0.582 \\
$F M_{2}$ & 7.61 & 8.534 & 0.608 & 0.578 \\
$F M_{3}$ & 5.6 & 5.097 & 0.607 & 0.604 \\
$F M_{4}$ & 5.58 & 6.097 & 0.712 & 0.702 \\
$F M_{5}$ & 4.8 & 4.672 & 0.766 & 0.760 \\
$F M_{6}$ & 4.81 & 4.723 & 0.768 & 0.752 \\
$F M_{7}$ & 4.8 & 5.382 & 0.694 & 0.686 \\
$F M_{8}$ & 5.58 & 6.065 & 0.703 & 0.683 \\
\hline$S(\theta)$ & 335.7 & 14.77 & \multicolumn{3}{|l}{} \\
\hline
\end{tabular}

Table 4: Initial and final estimates of $\theta$, plus statistics, after reparameterisation of the preexponential factor.

The reparameterisation does not change the problem, the sum of squares and the parameter values are unaffected. Only the confidence regions of the reparameterised parameters improve. Inspection of the singular values shows again that four of them are extremely small. The difference with the results from Table 3 is that now the last four columns of the matrix $V$ can be identified with the activation energies, $E_{i}$, i.e. the parameters which are the least well determined. This means that the longest principal axis of the elongated ellipse is rotated towards the $E_{i}$-axis after the reparameterisation. The singular values and the columns of matrix $V$ are shown in Figure 4.

The available measurements were carried out at temperatures between $323 \mathrm{~K}$ and $353 \mathrm{~K}$. Numerical experiments showed that we need a wider range of temperatures to estimate $E_{i}$ more accurately.

\section{Conclusions}

In this paper we presented a general approach for parameter estimation in dynamical systems, which are described by a set of differential algebraic equations. This approach has been applied to 


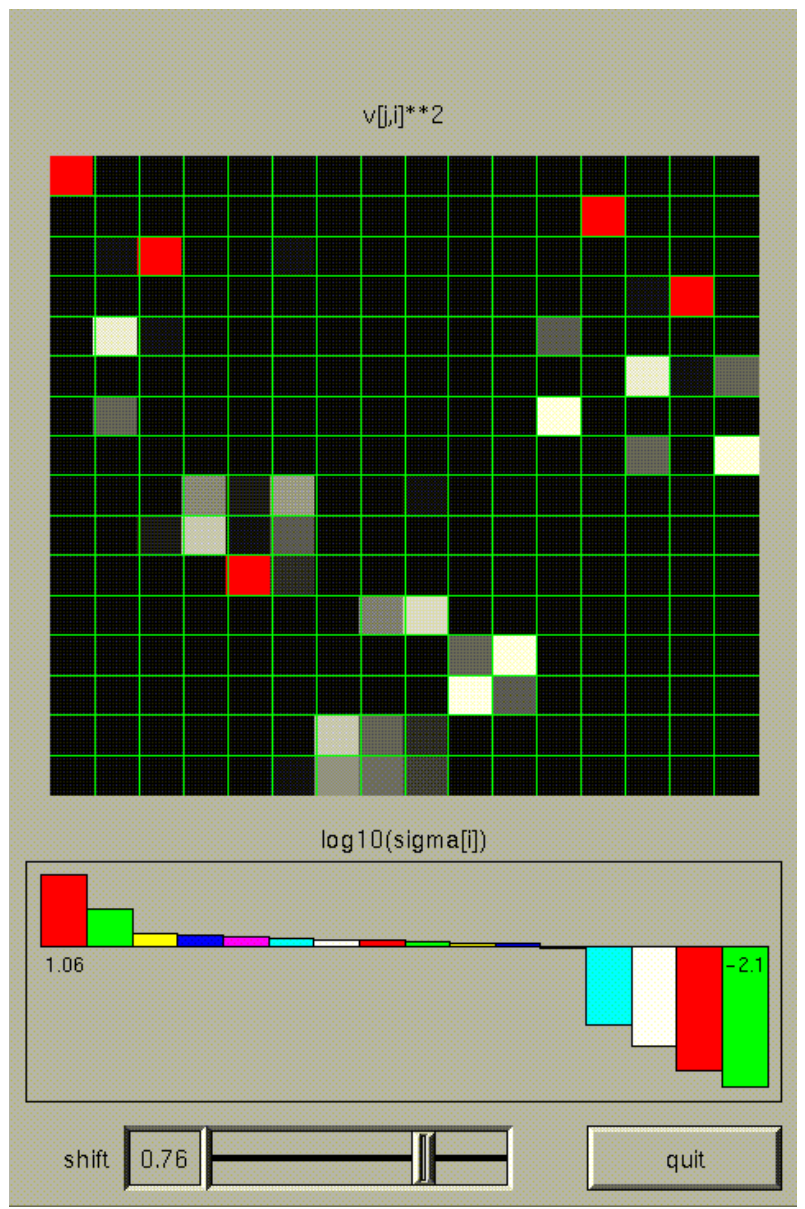

Figure 4: The squared entries of the matrix $V$ are matched on a grey scale (upper part). The black squares indicate small values and white squares represent values close to 1 . Each row of the matrix corresponds to a parameter. The logarithm of the corresponding singular values are plotted in the lower part.

a real life problem from reaction kinetics in order to estimate unknown reaction rates and unknown initial concentrations. The experiments were performed at different temperatures, which makes it necessary to use Arrhenius' law to derive the reactions rates. The unknown initial concentrations and pre-exponential factors could be estimated satisfactorily. For that purpose, however, we needed a reparameterisation of the pre-exponential factor. Due to the small range of the temperatures during the experiments it was not possible to estimate the activation energies with sufficient accuracy.

The strength of the method is the capability to decide for which parameters sufficient information is available in order to perform an accurate estimation. 


\section{References}

[Bar74] Y. Bard. Nonlinear Parameter Estimation. Academic Press, New York and London, 1974.

[BDB86] L.T. Biegler, J.J. Damiano, and G.E. Blau. Nonlinear parameter estimation: a case study comparison. AIChE Journal, 32(1):29-45, 1986.

[BW88] D.M. Bates and D.G. Watts. Nonlinear Regression Analysis and its Applications. John Wiley \& Sons, Inc., 1988.

[EHS95] C.T.H. Everaars, P.W. Hemker, and W. Stortelder. Manual of spIds, a software package for parameter identification in dynamic systems. Technical Report NM-R9521, CWI, 1995.

[Gea71] C.W. Gear. Numerical Initial Value Problems in Ordinary Differential Equations. Prentice-Hall, Inc. Englewood Cliff, NJ, 1971.

[GHW66] M. Gordon, A. Halliwell, and T. Wilson. Kinetics of the addition state in the melamineformaldehyde reaction. Journal of Applied Polymere Science, 10:1153-1170, 1966.

[Hem72] P.W. Hemker. Numerical methods for differential equations in system simulation and in parameter estimation. In H.C. Hemker and B. Hess, editors, Analysis and Simulation of Biochemical Systems, pages 59-80. North Holland Publ. Comp., 1972.

[HK93] P.W. Hemker and J. Kok. A project on parameter identification in reaction kinetics. Technical Report NM-R9301, CWI, 1993.

[Wat94] D.G. Watts. Estimating parameters in nonlinear rate equations. The Canadian Journal of Chemical Engineering, 72:701-710, 1994. 


\section{Appendix A. The Model File}

This appendix contains the model file as it was used as input for the parameter estimation program, spIds ([EHS95]). Comment lines start with a "\#".

The experimental data can be obtained via the author.

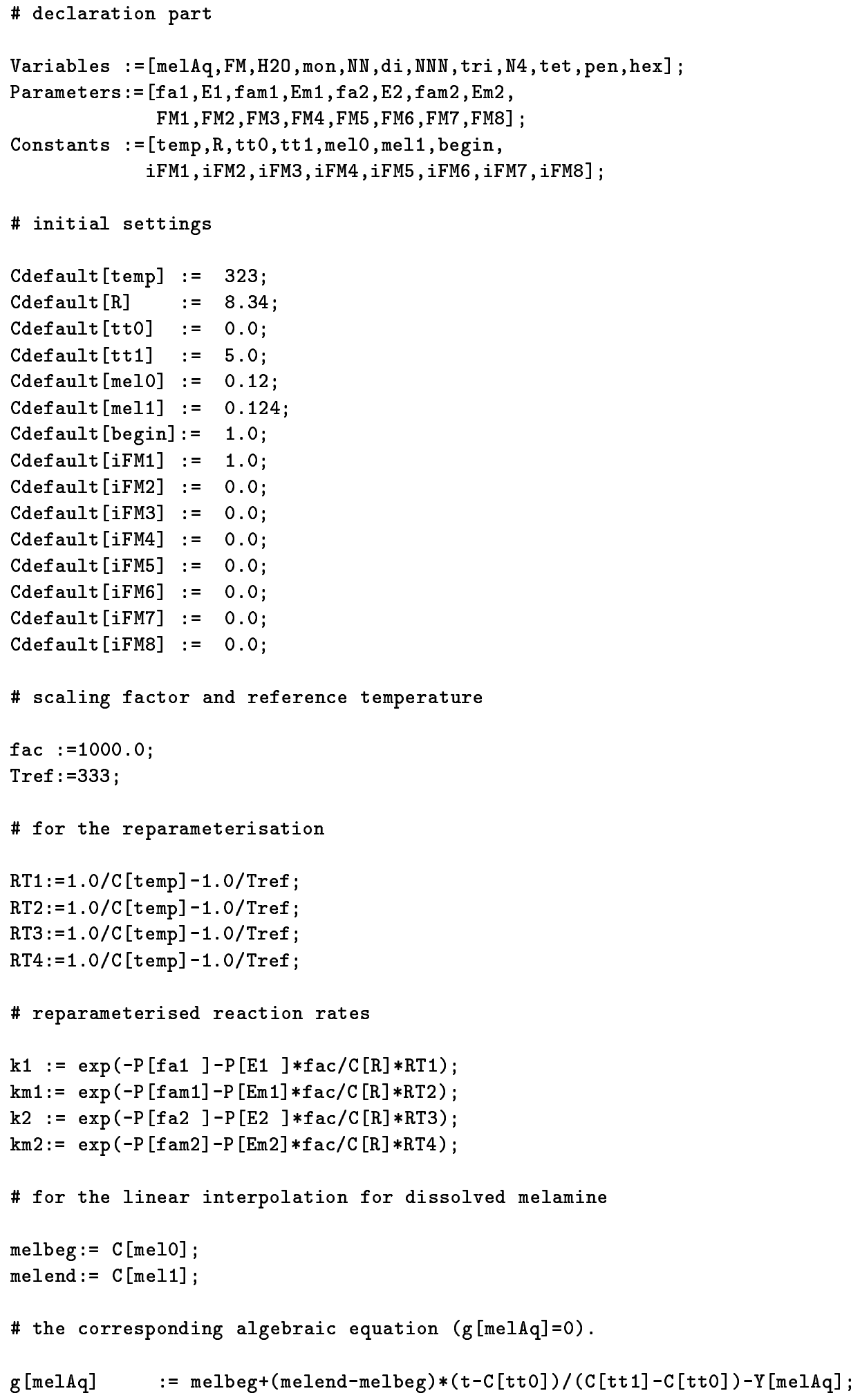


\# the differential equations

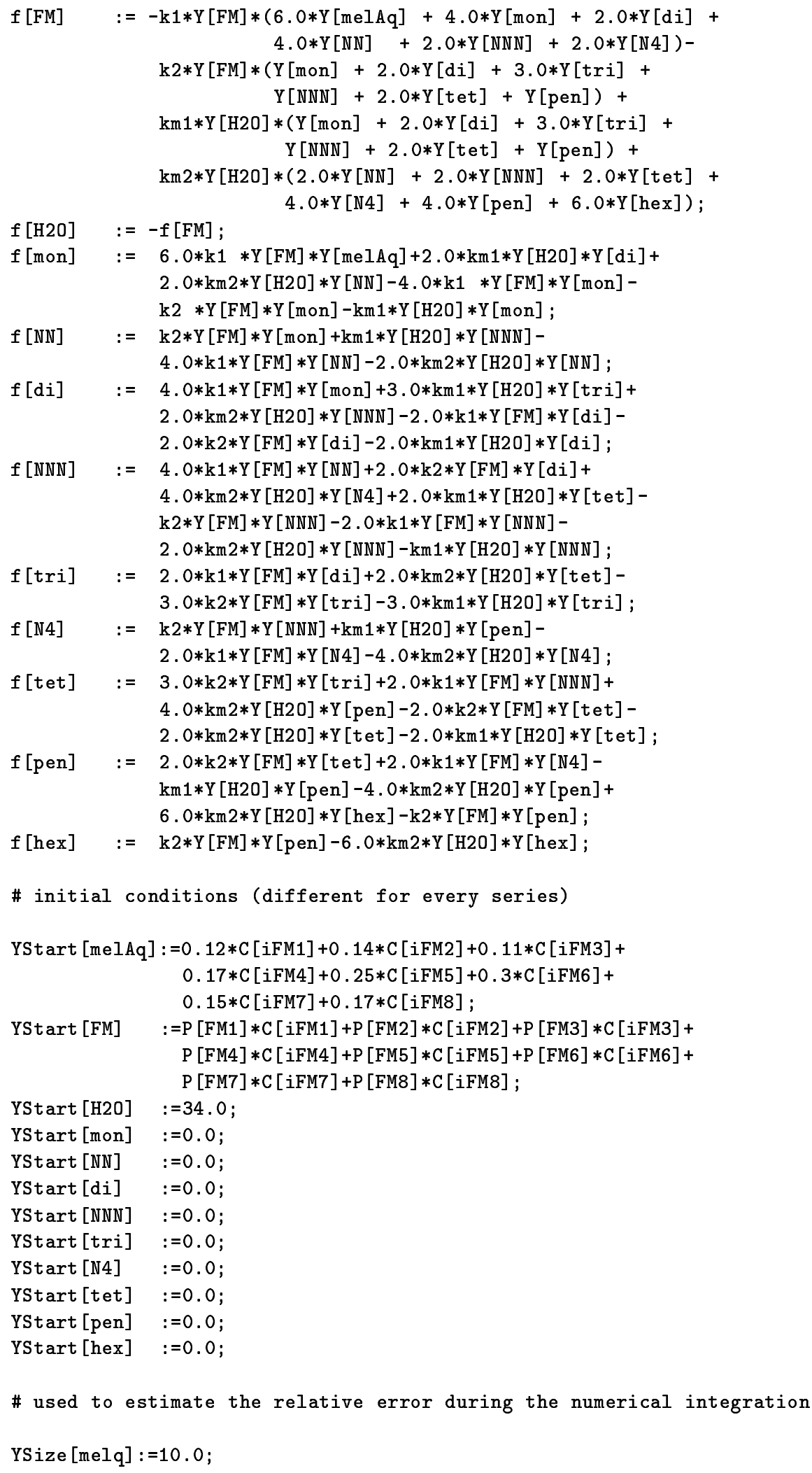




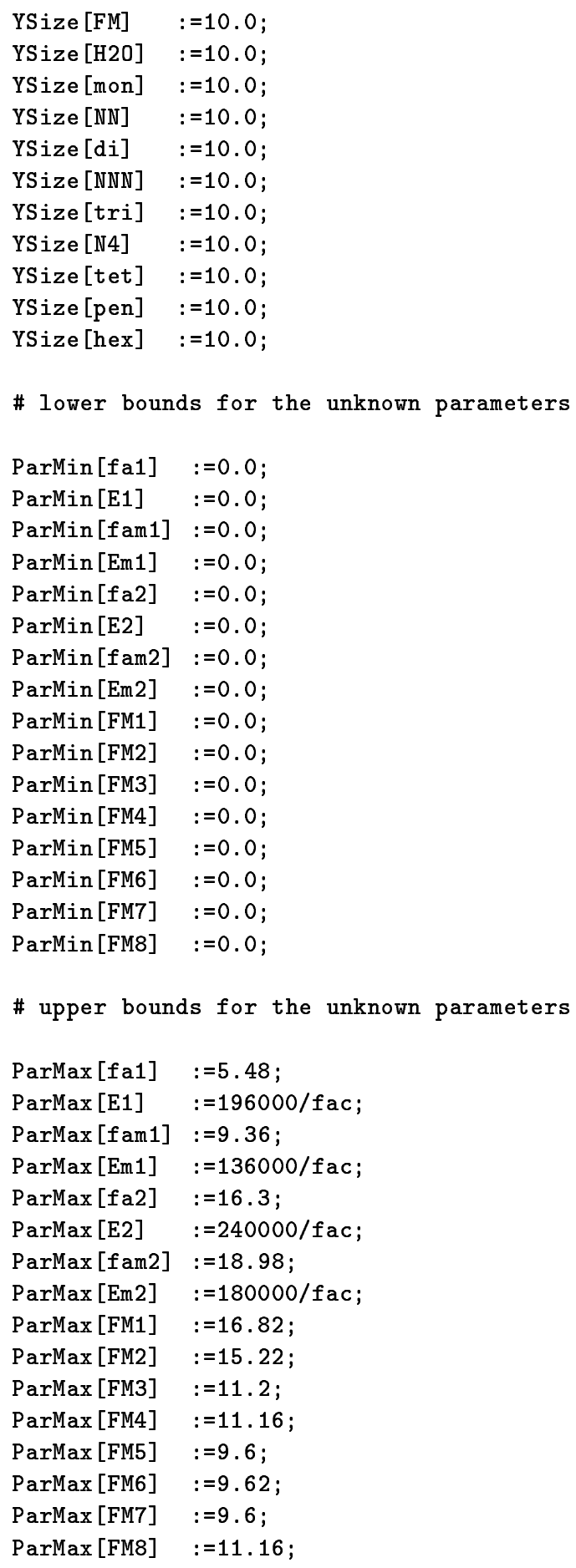

